1 Universidad de Buenos Aires (UBA) - Buenos Aires, Argentina. astolkiner@gmail.com

${ }^{2}$ Asociación Latinoamericana de Medicina Social (Alames)

- Buenos Aires, Argentina.

3 Universidad de El Salvador - San Salvador, El Salvador.

4 Universidade Estadual do Oeste do Paraná (Unioeste) - Cascavel(PR), Brasil.

\section{Lazos entre la Asociación Latinoamericana de Medicina Social y el Movimiento por la Salud de los Pueblos}

\author{
Bonds between the Latin American Social Medicine Association and \\ the People's Health Movement
}

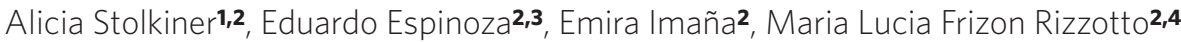

DOI: 10.1590/0103-11042020S112

\begin{abstract}
AL MOMENTO DE COMPARTIR ESTAS REFLEXIONES sobre las alianzas estratégicas que ha logrado el Movimiento por la Salud de los Pueblos (MSP) y la Asociación Latinoamericana de Medicina Social (Alames), no podemos obviar que estamos viviendo en América Latina intensos procesos políticos caracterizados por masivas movilizaciones populares de final abierto que confrontan con el regreso de mecanismos de represión y con golpes de Estado que recuerdan dolorosamente los métodos represivos y las rupturas institucionales de las últimas dictaduras. Alames se solidariza con la lucha de los pueblos y con las víctimas de la represión y urge a los gobiernos de la región a resolver democráticamente las legítimas demandas por más justicia social de los pueblos de nuestra región. Una confrontación de fondo en la cual la disputa entre la salud como derecho o la salud como mercancía no resulta ajena.

En este contexto, también, nos hemos visto profundamente dolidos por la noticia del fallecimiento del compañero David Sanders, fundador del MSP. David representaba en su propia figura los múltiples cruces que las trayectorias de ambas instituciones han tejido por casi dos décadas.

Alames surge a partir de núcleos que venían explorando la prolífica interacción entre ciencias sociales y salud, inspirada en los movimientos universitarios, campesinos y obreros de fin de los años 60 y comienzos de la década de los 70,que pronto se encontrarían enfrentadas a una serie de dictaduras militares y de gobiernos autoritarios que atravesaron la región. Nace en ese difícil contexto una asociación de fuerte identidad latinoamericana basada en una estrecha sinergia entre una producción científica y teórica de gran influencia en el pensamiento sanitario de la región y una práctica política situada en contextos complejos de fuertes desigualdades.

$\mathrm{Y}$ es que justamente un rasgo característico atribuido sin atenuantes a América Latina, es el de ser, sino la región mas pobre, sí la de ser la región más injusta e inequitativa del planeta.

También se percibía en otras partes del mundo una importantísima deuda sanitaria, que comenzaba a enfrentarse con experiencias de diferentes amplitud y profundidad, que encontraron resonancia en la declaración de Alma-Ata en 1978,la cuallanzó a nivel mundial la Atención Primaria de la Salud (APS) como una estrategia para alcanzar una mejora sustancial de la situación de las poblaciones, expresada en la idealista meta Salud para Todos.
\end{abstract}


Recogía y sistematizaba esta estrategia experiencias previas innovadoras que se habían vivido en diferentes partes del mundo, combinando saberes ancestrales, trabajadores comunitarios, tecnologías apropiadas y procesos sociales y políticos en regiones, países y comunidades muy distantes entre sí.

$\mathrm{Al}$ acercarse el fin del milenio crecía la convicción, en diferentes grupos, en el mundo sobre el incumplimiento de la meta Salud para Todos, una forma comunicacional de difundir los valores centrales que inscriben la salud en el plexo más amplio de los derechos humanos.

Es probablemente en la figura de Halfdan Mahler - Director de la Organización Mundial de la Salud (OMS) en el período en que la Reunión de Alma-Ata sucedió -, en quien se puede construir el eje para explicar este nuevo momento fundacional surgido en Bangladesh en Diciembre de 2000. 'A la Atención Primaria le fue mejor que a Salud para Todos', pues era una de las formas que con mayor frecuencia se elegía para denunciar la instrumentación de esta multiforme estrategia, que se había apenas ajustado a tibias reformas de los servicios de salud, pero que no había movido la aguja de los indicadores principales si de verificar resultados orientados a la equidad nos referimos.

No se trataba de ignorar aquellas mejoras demográficas en algunos países y regiones o un crecimiento significativo de lo que podría denominarse la primarización de los sistemas de salud con un primer nivel de mayor amplitud y capacidad resolutiva que se verificaba en el año 2000 y se verifica ahora. Se trataba más bien de recordar que la APS era una estrategia, una agenda, de reformas sociales, ambientales, políticas y sanitarias que hiciera del derecho a la salud una parte inseparable de un mundo más justo.

En una ponencia poco conocida de Mahler', de 2003, reafirma el camino original cuando expresa:

De ahí que yo en verdad crea que los valores fundamentales de la justicia social y la equidad son la esencia de la visión de Salud para Todos y de la Estrategia de Atención Primaria en Saludi(3-4).

Y también decía:

Usualmente se pregunta: '¿Podemos pagar el costo de la justicia social y la igualdad?' Yo propondría una contra pregunta: '¿Podemos sufragar el costo de la inestabilidad social y económica inherente a las tendencias actuales de maximizar las ganancias?'1(3-4).

Es probablemente esa convicción la que da origen, la que presta sentido, al movimiento por la salud de los pueblos. Una idea fundacional que hemos traducido en la simple frase que atribuimos al propio Mahler: 'Jamás el derecho a la salud va a ocurrir, ni se va a sostener si no deviene en una causa de los propios pueblos'. Será entonces durante la primera Asamblea por la Salud de los Pueblos en Bangladesh que un nuevo hecho político de alcance internacional va a conmover el ambiente sanitario mundial. Es la evidencia acumulada y política, magistralmente citada en la Declaración para la Salud de los Pueblos ${ }^{2}$ durante la primera Asamblea en 2000, así como las voces demandantes desde los pueblos crecientemente excluidos, los que configuran las declaraciones de Mahler que se adelantan a su tiempo:

Creo que es obvio que, si las tendencias actuales hacia la desigualdad continúan igual, nuestro mundo estará mucho más poblado, más contaminado, menos estable ecológicamente y mucho más vulnerable a la devastación económica, política y social. Creo que la transición más turbulenta estará asociada con el establecimiento de la equidad para todos los pobladores del planetai(4).

A pesar de la lejanía, este evento no podía pasar desapercibido para nuestra región, lo que se expresa en un gesto político contundente. Será entonces en la Ciudad de Cuenca, 
donde se va a celebrar la segunda Asamblea Mundial, probablemente en el escenario en el que se plasma la alianza estratégica de dos organizaciones iguales y distintas, hermanadas en la procura del derecho a la salud y con diferentes, aunque complementarias formas organizativas, estrategias, alianzas y formas de expresión política.

En la $2^{\text {a }}$ Asamblea Mundial del MSP en Cuenca se concreta, con base en los principios conceptuales, ideológicos y políticos, recogidos en la Declaración de Bangladesh/2000, los instrumentos organizativos fundamentales: El Movimiento de la Salud de los Pueblos (MSP/PHM) y la Universidad Internacional para la Salud de los Pueblos (Uisp/IPHU).

Por su parte, para el comienzo del milenio Alames había labrado una estrecha colaboración con la Asociación Internacional de Políticas de Salud (IAHP - por sus siglas en Inglés - al punto que sincroniza cada cuatro años sus congresos para consolidar su alianza. La IAHP traía una perspectiva predominantemente europea a los espacios de encuentro, mientras que el MSP había logrado captar, canalizar y expresar las principales preocupaciones de África, Asia y Medio Oriente.

El establecimiento de mecanismos de enlace, llevado adelante por compañeros de desde la Coordinación General de Alames, como Mauricio Torres, Rafael González, Mario Rovere y Alicia Stolkiner, se plasmó en diversos puntos y mecanismos de encuentro, sin embargo otros devenires jugaron y juegan para una mayor sensibilidad y confluencia de agenda.

Como ocurre en estos casos, el MSP fue creando su propia membresía en América Latina, lo que lejos de ser un obstáculo constituyó una oportunidad para establecer vínculos a escalas mas reducidas, generalmente expresando el color de las problemáticas nacionales o subnacionales.

La Medicina Social Latinoamericana no tuvo en gran estima la Atención Primaria tal como se implementó en muchos países de la región, entre otras cosas porque devino en un concepto extremadamente laxo, un ropaje apto para cualquier temporada, sin discriminar apropiadamente el carácter redistributivo o simplemente paliativo de diferentes intervenciones sanitarias, algunas complementarias, otras contradictorias que se desarrollaron bajo su nombre. Fue tan temprano como en 1985 que Mario Testa ${ }^{3}$ publicó su influyente trabajo 'Atención ¿̇Primaria o Primitiva? de Salud', que mantuvo el alerta por décadas sobre las formas concretas en que la APS se implementaba, aterrizada en medio de una epidemia de dictaduras.

Del mismo modo el MSP desarrolló un dispositivo crítico haciendo particular hincapié en la operación de la Fundación Rockefeller, apenas un año después de AlmaAta, en su centro de Bellagio Italia, instalando provechosamente el didáctico debate 'Atención Primaria de la Salud Selectiva vs. Comprensiva' que continúa siendo motivo de controversias y contribuye a tomar posición en este laxo campo.

La característica de los procesos políticos de la primera década del milenio, con once países en su apogeo por estar desalineados de las directivas de Washington, tuvo también una gran influencia. El Programa de Atención Básica, el movimiento de Mais Médicos y las Conferencias Sanitarias, consolidando una nueva etapa ahora municipalizada, del Sistema Único de Salud de Brasil, las Reformas Sanitarias de El Salvador, de Ecuador, o de Paraguay, el programa Barrio Adentro de Venezuela, la Escuela Latinoamericana de Educación Médica (Elam) en Cuba, formando profesionales de salud de todos los países con una fuerte orientación a la Medicina General.

Pero, quizás, la construcción relativamente reciente que generó mayor identidad a la región, tanto en el MSP como en Alames, ha sido el surgimiento del concepto del Sumak Kawsay del Buen Vivir que probablemente tenga más influencia que en el Ecuador y el notable proceso político, social, económico y cultural que ha protagonizado el actual Estado Plurinacional de Bolivia. 
El reconocimiento de una nueva identidad indo-afro-latinoamericana surge como expresión de culturas reprimidas y silenciadas por décadas que requieren nuevos dispositivos de encuentro, de dialogo, para alcanzar a pueblos originarios, sin mensajes, encubiertamente colonizados. La interculturalidad política que exige la valoración y el respeto recíproco de las culturas que dialogan, que entran en contacto, ha descubierto un concepto revelador de una forma diferente de relación con la naturaleza y con la vida en comunidad.

No es el motivo de este breve informe, pero para expresar en una frase el potencial de transformación de este concepto permítasenos contrastar el mandato judeo-cristiano predominante en occidente: 'dominarás la tierra' con el mandato de los pueblos ancestrales 'la tierra no nos pertenece, nosotros pertenecemos a la tierra'. Una diferencia que adquiere un tono de dramatismo en contextos en que la viabilidad misma de la vida en nuestro planeta está amenazada.

La Conferencia de Astaná sobre Atención Primaria, a 40 años de Alma-Ata, en octubre del 2018, resultó un nuevo motivo de encuentro, de unidad en la diversidad.

Comenzando por las diferencias que probablemente quedarán pobremente expresadas en el par incidencia confrontativa o incidencia colaborativa. Alames ha elegido con frecuencia una estrategia de incidencia confrontativa con la OMS, -como se vio en los fuertes debates sobre determinación o determinantes sociales de la salud, reaccionando frente al proceso del mal llamado 'Informe Marmot' que ignoró 25 años de producción latinoamericana.

El MSP, por su parte, probablemente por la fortaleza de contar en sus filas con un ex Director de la OMS, ha elegido una estrategia de mayor cercanía intentando forzar a la OMS a una cierta rendición de cuentas (accountability) y tratando de incidir a través de documentados instrumentos como el seguimiento de Conferencias Sanitarias y las reuniones o la publicación del Global Health
Watch (Observatorio de la Salud Global).

Frente al intento de la OMS, del Banco Mundial, del Gobierno del Kazajistán, de reeditar una reunión que saldara las controversias -justamente a un año del fallecimiento de Mahler-, emergió nuevamente el reflejo de un posicionamiento crítico que tuvo como punto de máxima convergencia la declaración desde los documentos preparatorios de intentar subsumir la Atención Primaria a la controversial Cobertura Universal de Salud (CUS).

Pero aquí nuevamente se perciben las diferencias y complementaciones, ya que Alames publica un documento crítico ya desde antes que la reunión ocurriera, a partir de los documentos provisorios que la propia OMS, mientras que el MSP logra participar de la reunión, matizando a través de los mismos debates su posicionamiento crítico.

El desafío para los próximos años es el de cómo profundizar la agenda compartida, cómo multiplicar los encuentros y los militantes involucrados en este dialogo que trae la riqueza de pueblos de todo el mundo. Pueblos que somos tan diferentes en nuestras lenguas, en nuestras costumbres, en nuestras culturas, pero que compartimos décadas sino siglos de resistencia a un capitalismo transnacionalizado, depredador y extractivista, que procura a través de una globalización engañosa arrasar con nuestras identidades, imponiendo sus modelos de consumo.

Los derechos de la madre tierra, los derechos de los migrantes internos y externos, de los refugiados bélicos y ambientales, la lucha internacional contra el patriarcado, el movimiento de jóvenes contra el cambio climático y la necesidad de una fuerte descolonización de nuestras sociedades y de nuestros gobiernos, nos sigue convocando para garantizar el derecho a la salud.

La entrega generosa, la actitud abierta de comprensión más allá de cualquier barrera cultural, la afabilidad y el testimonio de vida de David Sanders, son un motivo más para enriquecer nuestra agenda, potenciar 
nuestras estrategias y acrecentar los lazos de hermandad entre Alames y el MSP.

\section{Agradecimientos}

Agradecemos a Mario Rovere por su contribución en la construcción y revisión final del texto.

\section{Colaboradores}

Stolkiner AI (0000-0001-9372-7556)*, Espinoza E (0000-0002-4894-2924)*, Imaña E (0000-0001-9895-2445)*, Rizzotto MLF (0000-0003-3152-1362)* tuvieron roles iguales en la elaboración del artículo.

\section{Referencias}

1. Mahler H.Liderazgo y Equidad en Salud, Conferencia inaugural del Centro de Investigación y Desarrollo en Salud. El Salvador: Universidad de El Salvador; 2003

2. People's Health Movement. Declaración para la Salud de los Pueblos [internet]. 2000. [acceso en 2019 nov 1]. Disponible en: file://D:/Revista/ Downloads/2000-M1-Declaracion_para_la_Salud_ de_los_Pueblos.pdf.
3. Testa M. Atención primaria o primitiva de salud. Cuad. Médico Soc. [internet] 1985. [acceso en 2019 nov 1]; (34):1-8. Disponible en: http://www.trabajosocial.unlp.edu.ar/uploads/docs/testa_mario_atencion_primaria_o_primitiva_de_salud.pdf.

Recibido el 13/01/2020

Aprobado el 13/01/2020

Conflicto de intereses: inexistente

Apoyo financiero: no hubo 